\title{
Long-Term Objective Physical Activity Measurements using a Wireless Accelerometer Following Minimally Invasive Transforaminal Interbody Fusion Surgery
}

\author{
Kevin Phan, Ralph J. Mobbs \\ Neuro Spine Clinic, Suite 7a, Level 7 Prince of Wales Private Hospital, Randwick, Australia
}

\begin{abstract}
We report on a case of a patient who underwent minimally invasive transforaminal lumbar interbody fusion (mi-TLIF) with objective physical activity measurements performed preoperatively and postoperatively at up to 12-months using wireless accelerometer technology. In the first postoperative month following surgery, the patient had reduced mobility, taking 2,397 steps over a distance of $1.8 \mathrm{~km}$ per day. However, the number of steps taken and distance travelled per day had returned to baseline levels by the second postoperative month. At one-year follow-up, the patient averaged 5,095 steps per day in the month over a distance of $3.8 \mathrm{~km}$; this was a $60 \%$ improvement in both steps taken and distance travelled compared to the preoperative status. The use of wireless accelerometers is feasible in obtaining objective physical activity measurements before and after lumbar interbody fusion and may be applicable to other related spinal surgeries as well.
\end{abstract}

Keywords: Accelerometer; Fitbit; Fusion; Transforaminal interbody fusion surgery; Physical activity; Objective measurement

\section{Introduction}

The costs of delivery of surgical care for spinal pathologies are significant $[1,2]$ and there is a need to provide objective measurements to monitor mobility and motor recovery and management of symptoms [3]. Optimal monitoring presurgery and postsurgery is important not only from the point-of-view of patients and healthcare professionals, but also from a societal and economic perspective. In addition to monitoring the safety and efficacy of surgical procedures, reports on discharge disposition and functional recovery are significant for optimal health care delivery and resource allocation [4].
The measurement and comparison of recovery and functional outcomes following spine surgery has traditionally been quantified using subjective rating systems for symptoms and quality of life. These subjective measures include the visual analog scores (VAS) for back and leg pain, Oswestry disability index (ODI) and Short Form Health survey scores for comparing preoperative and postoperative conditions to gauge improvement in a functional outcome. However, the major pitfall with these measures is their subjective nature and the inherent bias with personal evaluation. Indeed, self-rated scores are influenced by the patients' perception of their symptoms and disability [5].

\footnotetext{
Received May 21, 2015; Revised Jun 6, -2015; Accepted Jun 7, 2015

Corresponding author: Kevin Phan

Neuro Spine Clinic, Suite 7a, Level 7 Prince of Wales Private Hospital,

Barker Street, Randwick, New South Wales 2031, Australia

Tel: +61-2-9650-4766, Fax: +61-9680-4943, E-mail: kphan.vc@gmail.com
} 
With the advent of wireless accelerometers, we have the capability to record real-time data regarding movement and function and these include the number of such steps taken and the distance travelled [6]. The potential advantage of these accelerometers in the setting of spine surgery is the ability to provide continuous objective reads of various physical parameters that define ambulatory function, activity levels and extent of recovery. This is in contrast to the traditional subjective scores for pain and function that depend on personal perception.

This case report discusses the use of wireless accelerometer technology in a patient who underwent minimally invasive transforaminal lumbar interbody fusion (mi-TLIF) to objectively measure mobility and function preoperatively and postoperatively up to 12-months follow-up.

\section{Case Report}

A 60-year-old male presented with severe discogenic low back pain and bilateral radiculopathy, worsening over a period of 7 years. These symptoms had a significant impact on his daily routine and capacity to work as a vehicle mechanic. His medical history included ischaemic heart disease with a cardiac stent, thus requiring him to maintain regular exercise for his general health.

The patient had experienced only limited pain relief with conservative treatments that included physical therapy, epidural steroid injections, non-steroidal anti-inflammatory medications and opiates. He was then qualified for a 2-level mi-TLIF operation with interbody grafting and ES2 percutaneous pedicle screw fixation (ES2, STRYKER Instruments, Kalamazoo MI, USA). The patient's steps and distance travelled were monitored for 1 month preoperatively and subsequently over a period of 12-months, using a Fitbit accelerometer (Fitbit Inc., San Francisco, CA, USA). Fitbit accelerometer is commonly used for objective measurements of physical activity. It detects and records the number of steps taken, intensity of physical activity, duration, distance travelled and estimated caloric expenditure using accelerometer technology, which can be adjusted according to user's age, sex, height and weight. The Fitbit was worn during all waking hours and not during sleep. For comparison, preoperative and postoperative functional scores were also recorded, including VAS back, VAS leg, ODI and short form (SF)-36 mental composite score (MCS) and physical composite score (PCS) scores.

The operation proceeded as planned and there were no perioperative complications noted (Fig. 1). He was discharged on day 3 postoperation, and returned to light duties after 4 weeks. Fitbit data in the month prior to operation showed that the patient had taken an average of 3,249 steps per day, over an average daily distance of $2.4 \mathrm{~km}$. In
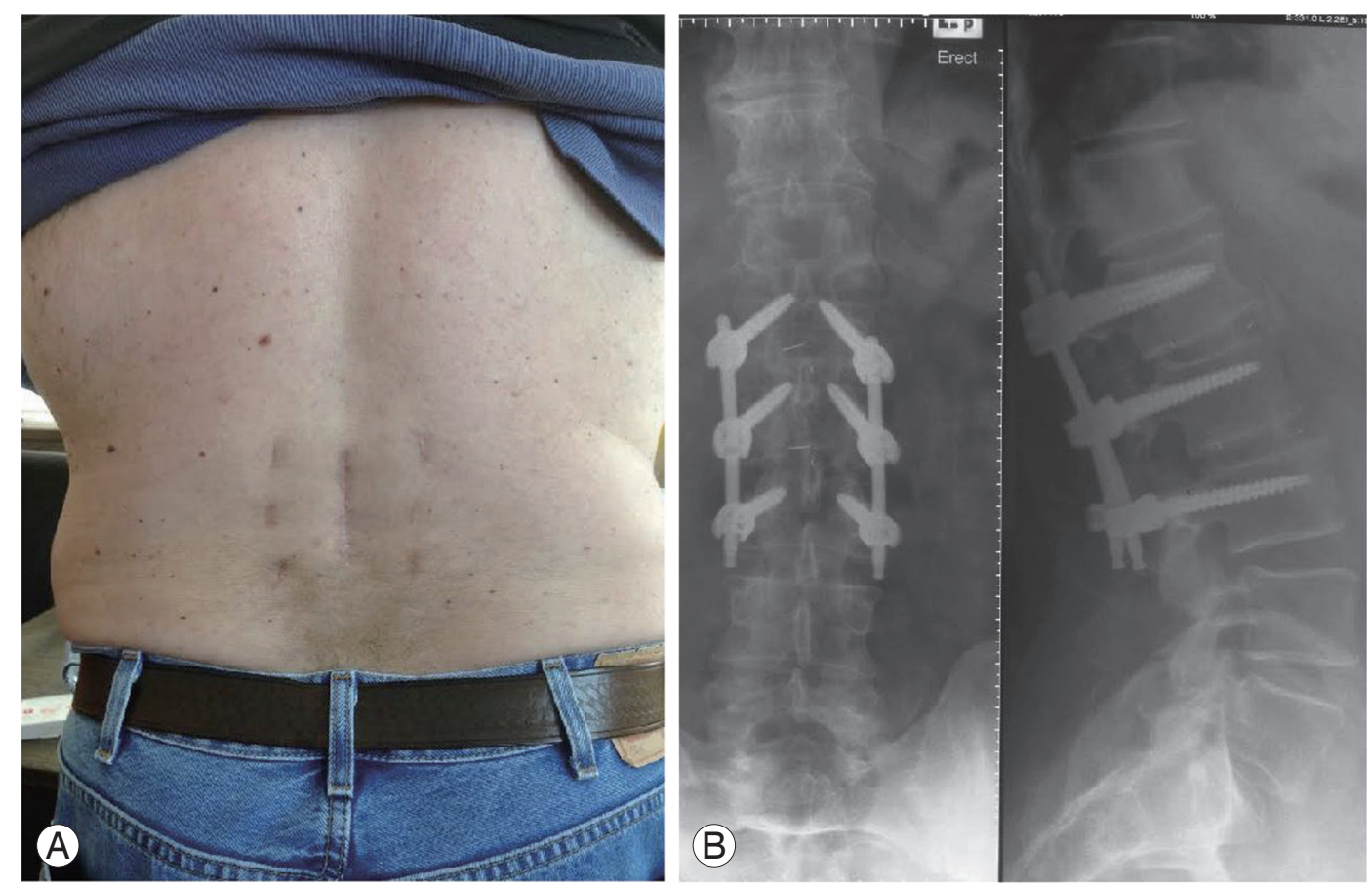

Fig. 1. Postoperative outcome. (A) Incision profile. (B) Standing X-rays at 3 months postoperative. 
the first postoperative month following surgery, the patient had reduced mobility, taking 2,397 steps over $1.8 \mathrm{~km}$ per day. However, the number of steps taken and distance travelled per day had returned to baseline levels by the second postoperative month and continued to increase thereafter (Fig. 2). At one-year follow-up, the patient averaged 5,095 steps per day for a distance of $3.8 \mathrm{~km}$; this was a $60 \%$ improvement in both steps taken and distance travelled compared to preoperation numbers (Fig. 3 ) and the operation was deemed a success.

Overall, there was significant improvement in VAS back and leg pain scores, ODI, and SF-12 ratings for the patient at follow-up compared to baseline. VAS back pain scores reduced from 8.0 to 1.0 over 12-months, while VAS leg pain scores reduced from 4.0 to 1.0 units. Postoperative ODI score was 6.7 compared to preoperative ODI of 53.3. As such, there was significant correlation between changes in ODI score and improvement in physical activity

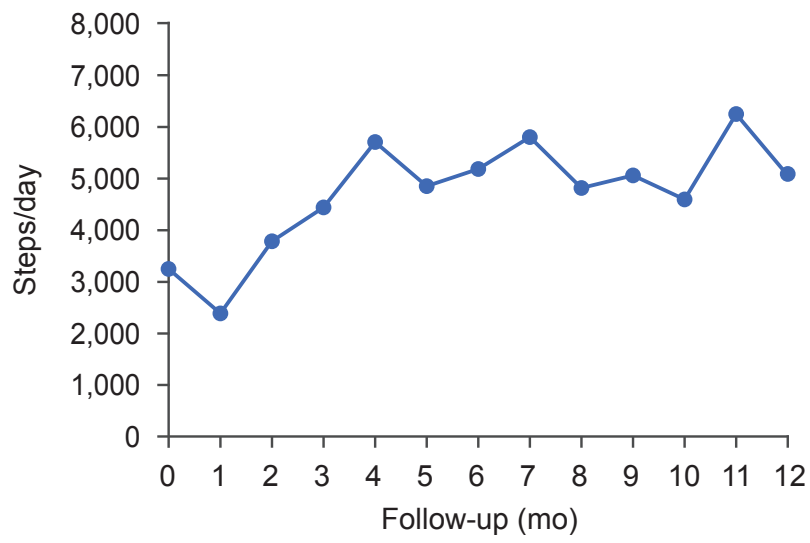

Fig. 2. Average steps per day at monthly follow-up.

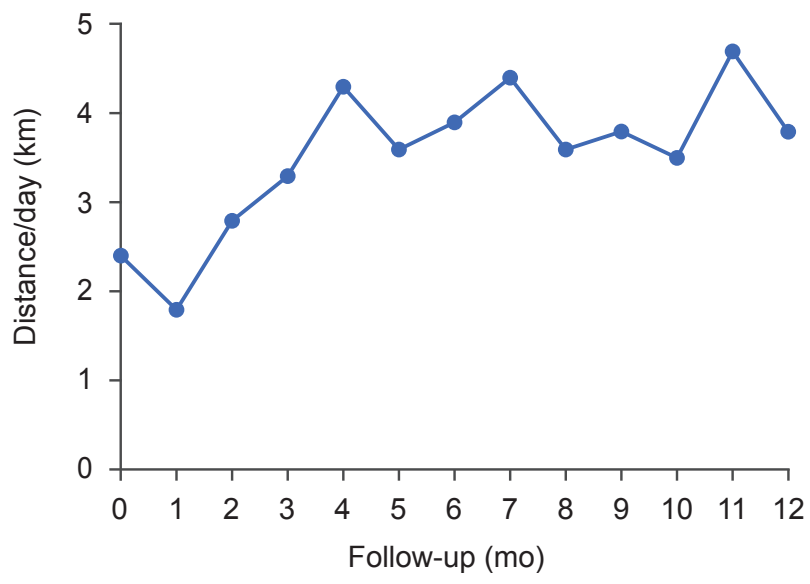

Fig. 3. Average distance travelled per day at monthly follow-up. at follow-up as quantified by the accelerometer. PCS-36 scores increased from 32.6 to 53.1, whilst MCS-36 scores changed by a small margin, from 50.0 to 59.9 .

\section{Discussion}

Functional recovery following spine surgery has traditionally been reported via surrogate parameters such as duration of hospital stay along with quality of life, pain and functional scores such as VAS, SF12/SF36, Roland Morris disability index and ODI. The main disadvantages with these approaches include the inherent bias and individual variation in assessment with self-evaluation as well as a lack of standardization of outcomes across different surgical procedures [7,8]. Mental state, depression, psychological factors and the role of compensation and litigious action, well outside the scope of a surgical intervention, can result in significant variances of these Quality of Life outcome scores [9]. Advances in wireless technology coupled to accelerometers and global positioning systems have led to the advent of real-time recording and monitoring with the potential of providing objective quantification of activity and function during the postoperative recovery period. Whilst there have been several pilot studies reporting the use of accelerometers for evaluating function and recovery [10-12], there have only been few instances where this technology has been applied to spine surgery. In our report we provide one such example with an example of mi-TLIF coupled to a wireless functional reporting technology.

In the present case, postoperative function with oneyear follow-up was monitored using objective accelerometer measurements as well as using subjective self-report scoring systems (VAS, ODI, SF-36). Compared to his physical activity function prior to surgery, the patient had a $60 \%$ improvement in the number of steps taken and distance walked per day at the one-year follow-up,. The objective physical activity data from the Fitbit accelerometer was compared to the subjective self-report scores in VAS leg pain, ODI scores and PCS-36 scores and both showed significant improvements.

The accelerometer data showed a reduction in both the steps taken and distance travelled within the first month following surgery. This is not surprising given that the patient is often hospitalized coupled with limited movement and physical activity during the early phase of postoperative recovery. Return to baseline preoperative 
physical activity occurred within 2 months, and physical activity almost doubled in the 4th month postoperation. This data suggests that if self-reported scores are predictive of recovery and function, any improvements in such scores would only likely to be observed if the patients are surveyed at 4 months and more postoperation.

In summary, we present the case of a patient who underwent mi-TLIF with excellent recovery and improvement in physical activity and symptoms demonstrated by both the objective accelerometer data and the selfreported subjective index scores. The use of accelerometers for objective measurements of physical activity after spine surgery is feasible, and their use and benefit should be validated in larger prospective studies.

\section{Conflict of Interest}

No potential conflict of interest relevant to this article was reported.

\section{References}

1. Deyo RA, Gray DT, Kreuter W, Mirza S, Martin BI. United States trends in lumbar fusion surgery for degenerative conditions. Spine (Phila Pa 1976) 2005;30:1441-5.

2. Weinstein JN, Lurie JD, Olson PR, Bronner KK, Fisher ES. United States' trends and regional variations in lumbar spine surgery: 1992-2003. Spine (Phila Pa 1976) 2006;31:2707-14.

3. Pryce R, Johnson M, Goytan M, Passmore S, Berrington N, Kriellaars D. Relationship between ambulatory performance and self-rated disability in patients with lumbar spinal stenosis. Spine (Phila Pa 1976) 2012;37:1316-23.

4. Soegaard R, Christensen FB. Health economic evaluation in lumbar spinal fusion: a systematic literature review anno 2005. Eur Spine J 2006;15:1165-73.

5. Iversen MD, Katz JN. Examination findings and selfreported walking capacity in patients with lumbar spinal stenosis. Phys Ther 2001;81:1296-306.

6. Rand D, Eng JJ, Tang PF, Jeng JS, Hung C. How active are people with stroke?: use of accelerometers to assess physical activity. Stroke 2009;40:163-8.

7. Tudor-Locke CE, Myers AM. Challenges and opportunities for measuring physical activity in sedentary adults. Sports Med 2001;31:91-100.

8. Bassett DR Jr, Cureton AL, Ainsworth BE. Measurement of daily walking distance-questionnaire versus pedometer. Med Sci Sports Exerc 2000;32:1018-23.

9. Mobbs RJ, Gollapudi PR, Chandran NK. Outcome following anterior cervical discectomy in compensation patients. J Clin Neurosci 2001;8:124-5.

10. Culhane KM, Lyons GM, Hilton D, Grace PA, Lyons D. Long-term mobility monitoring of older adults using accelerometers in a clinical environment. Clin Rehabil 2004;18:335-43.

11. Dalton A, Khalil H, Busse M, Rosser A, van Deursen R, Olaighin G. Analysis of gait and balance through a single triaxial accelerometer in presymptomatic and symptomatic Huntington's disease. Gait Posture 2013;37:49-54.

12. Sumukadas D, Laidlaw S, Witham MD. Using the RT3 accelerometer to measure everyday activity in functionally impaired older people. Aging Clin Exp Res 2008;20:15-8. 